\title{
La congelación de óvulos en el ámbito laboral por causas sociales: nueva estrategia empresarial para controlar el cuerpo de la mujer
}

\author{
The Freezing of Eggs by Social Causes: \\ New Market Strategy for Women Body Control
}

\section{RESUMEN}

En España diversas empresas promocionan la congelación de los óvulos entre sus trabajadoras para que la maternidad no sea una dificultad a la hora de desarrollar la carrera profesional de las mujeres. Esto no es sino la enésima estrategia que el sector económico ofrece del control del cuerpo femenino para favorecer los intereses de las organizaciones, bajo la excusa de ser una herramienta que favorece la libertad de sus empleadas a la hora de trazar su itinerario personal y laboral. Una iniciativa más que muestra la maternidad como un freno -ya que los cuidados se asocian directamente a las mujeres- y que se traduce en el envejecimiento sin relevo de la población española, en vez de buscar alternativas que favorezcan la conciliación y que sin duda beneficiarían a la sociedad en su conjunto y fomentarían una corresponsabilidad más real y comprometida.

Palabras clave: maternidad, conciliación, economía, cuerpo, mercantilización.

\section{Abstract}

In Spain several companies promoted the freezing of eggs among their workers so that motherhood is not a difficulty in developing women's professional career. This is the thousandth strategy that the economic sector provides in order to control women's body for the interests of the organizations, under the excuse of being a tool that promotes freedom of their employees, when they have to draw up their personal itinerary and labour. Another initiative that show motherhood as a brake -as care are directly associated with womenand that translates into aging without relief of the Spanish population, instead of look for alternatives that would favour the conciliation and benefit the society as a whole and foster a more real and committed stewardship.

Keywords: Motherhood, conciliation, economy, body, commercialization.

\section{SUMARIO}

1.- Introducción. 2.- Efectos de la maternidad para las mujeres en el ámbito empresarial. 3.- Dificultades y soluciones para favorecer la conciliación. - Conclusiones. - Bibliografía. 


\section{Introducción}

En octubre de 2014 distintas empresas de Silicon Valley -tales como Facebook ${ }^{2}$, Google, Yahoo, Uber, Spotify y Apple- empezaron a financiar la congelación de los óvulos de sus empleadas, con la idea de favorecer el desarrollo profesional de éstas y permitirles conciliar una vida familiar plena. De este modo, el factor biológico que perjudicaba a la fertilidad quedaba subsanado y ellas podían cumplir el sueño de ser madres en el momento personal y laboral más propicio.

Esta iniciativa ha generado polémica: si bien desde el lado empresarial se podría enmarcar como un ejemplo más de política de responsabilidad social -al igual que contribuyen con otros beneficios sociales para con sus trabajadores con la idea de desterrar la visión del empleador como depredador del talento y esfuerzo de sus empleadas y empleados- desde la perspectiva de género se observa una nueva manera de control del cuerpo de las mujeres y una intromisión en su intimidad bajo el supuesto paraguas de su libertad de elección, que no es sino una nueva estrategia de expolio de la capacidad productiva de éstas bajo la maquinaria empresarial y el capitalismo, una invitación implícita a retrasar la maternidad. Asimismo, se refuerza la idea de que la maternidad es un problema en el mercado de trabajo para las mujeres, tanto a la hora de mantener el empleo como en la promoción interna de éstas.

Ahora, esta iniciativa ha llegado a España hace unos meses. Concretamente, en noviembre de 2017 las empresas valencianas Caixa Popular, Nunsys, Bodegas Vicente Gandía y Arroz Dacsa -que forman parte del Club de las Primeras Marcas de la Comunidad Valenciana ${ }^{3}$ - han aplicado de manera pionera la medida ya puesta en marcha en Estados Unidos. Así, se comprometen a sufragar un 10\% de los tratamientos de vitrificación de óvulos de sus trabajadoras o de las parejas de sus trabajadores tras la firma de un convenio con el Instituto Valenciano de Infertilidad (IVI) para promocionar esta práctica, que permite conservar los óvulos, con un coste medio de 2500 euros para cuatro años, según fuentes de la institución médica.

Al respecto, el presidente del Club de las Primeras Marcas de la Comunidad Valenciana, Juan Manuel Baixauli, señala en una noticia publicada en el periódico $E l$ Mundo en 2017 que «el 38.1\% de las mujeres considera que tener un hijo entorpece su carrera profesional», por lo que retrasan su maternidad, y vaticina que en un futuro hacer uso de este recurso "será algo habitual y muy valorado por todas las partes involucradas. Es simplemente una opción que ofrecemos a nuestra plantilla y creemos que con ello contribuimos a vencer uno de los mayores frenos a la maternidad».

2 La entonces directora de operaciones de Facebook, Sheryl Sandberg, explicó que esta decisión fue provocada por la petición de una empleada con cáncer que no podía pagarse un tratamiento, que tiene un coste medio de 10000 dólares. A partir de entonces, otras grandes empresas del sector tecnológico copiaron esta iniciativa.

3 En estos momentos el Club de las Primeras Marcas de la Comunidad Valenciana tiene 22 empresas reconocidas, que supone un volumen de facturación total de unos 4000 millones de euros anuales y cuentan con 25000 empleados. Información disponible en: http://clubdeprimerasmarcas.com/ (Fecha de consulta: 15/03/18). 
José Remohí -fundador del IVI- considera en dicha pieza informativa que «dentro de 20 años todas las mujeres lo harán, todas» porque la edad media para tener hijos avanza, algo que puede llegar a ser «un problema grave reproductivamente». Además, el profesor apunta que congelar óvulos «se convertirá en una necesidad social y la Seguridad Social lo cubrirá» (Europa Press, 2017).

En nuestro país, el retraso de la maternidad se hace mayor con el paso de las décadas y, simultáneamente, se reducen el número de hijos e hijas, lo que se refleja en un desarrollo negativo de la curva demográfica. Así, en julio de 2017 ya se registraban de manera provisional -según datos del INE- 32.000 muertes más que nacimientos. Concretamente, frente a las 218.688 personas fallecidas, solo habían nacido 186.783 bebés, lo que se traduce en una sociedad envejecida.

Junto con esto, se observa según datos del INE (2017) $)^{4}$ en cuanto a indicadores de fecundidad, como desde hace casi 40 años, el nacimiento del primer hijo o hija se ha ido retrasando progresivamente. Concretamente, en 1980 la media de edad de las mujeres para convertirse en madres era de 25,0562 años; en 1990 pasa a ser de 26,805862; en 2000 es de 29,090763 años; 2010 se establece en 29,824204 y 2017 alcanza los 30,896107. Así, se observa como con la recta final del siglo XX se consolida una tendencia al alza en el retraso del nacimiento de hijos e hijas que no ha sufrido cambios hasta la fecha.

Con este contexto, medidas como la anteriormente citada, contribuyen a favorecer más si cabe esa diferencia notable en ambas partes de la campana demográfica de nuestro país, en vez de apostar por políticas realmente conciliadoras y de corresponsabilidad con un espectro general y en todos los ámbitos.

Esta iniciativa de congelación de óvulos se ha lanzado como una nueva manera de empoderar a las mujeres y se ha comparado con la libertad sexual que lograron cuando se democratizó el uso de anticonceptivos y como otro elemento más que ayude a la conciliación, cuando esta iniciativa -promocionada por el sector empresarial- beneficia a las empresas mediante el control de los cuerpos de las mujeres, no a las trabajadoras.

Asimismo, desde el ámbito jurídico se podría plantear si esta medida es inconstitucional, ya que si bien en una entrevista laboral no se le puede preguntar a una mujer o a un hombre sobre su estado civil ni los planes de formar una familia - porque supone vulnerar el artículo 14 de la Constitución Española-, indagar e invadir la privacidad de las trabajadoras viola dicha norma, además de perpetuar los estereotipos sobre las mujeres.

$\mathrm{Y}$ es que fomentar la maternidad sin renunciar a la vida profesional no pasa por retrasar la primera con la congelación de embriones. Este modo de maternidad extendida se interpreta más que como un empujón para la natalidad, como una invitación para dar un mayor rendimiento en el trabajo, cuando a lo que realmente se procede es a la mercantilización de la reproducción humana. Al respecto, Nuño recuerda como:

4 Se ha seleccionado toda la muestra de mujeres del territorio nacional entre los años 1980 y 2017, y solo se ha escogido la edad que tenían de media en el nacimiento del primer hijo o hija. 
Los cuerpos de las mujeres han sido, históricamente, objeto del ejercicio del poder patriarcal. Todas las culturas, en mayor o menor medida, promueven las condiciones materiales y simbólicas necesarias para reforzar su sumisión y dependencia social, y, con ello, garantizar que estén «a mano para el uso sexual o reproductivo». Cada sociedad, en función de sus necesidades o intereses, no ahorra esfuerzos en inocular o reprimir el deseo reproductor (Nuño, 2016: 685).

Por su parte, Guerra critica duramente el fomento de la congelación de óvulos por parte de las empresas entre sus empleadas y se pregunta si:

En el fragor de una agotadora y exigente carrera profesional ¿por qué no «externalizar» también en el futuro la gestación tan denostada por los mercados de trabajo capitalista? Las lógicas de la servidumbre [...] se imponen a las clases más desfavorecidas y feminizadas en la actual fase del capitalismo. Las cadenas globales de cuidados protagonizadas por las mujeres migrantes, los mercados prostitucionales en los países desarrollados y en el turismo sexual alimentados por las redes de trata de mujeres transnacionales y, ahora, el negocio de la gestación subrogada parecen obedecer a esta degradante tendencia en la que el capitalismo global refuerza al patriarcado y viceversa (Guerra, 2017: 41).

En esta misma línea, Nuño (2016: 688) resalta que «a lo que nos enfrentamos no es solo a una nueva forma de mercantilizar el cuerpo de las mujeres sino, a su vez, a un nuevo modo de producción que pone a disposición del libre mercado seres humanos gracias a la "fuerza productiva" de miles de úteros: el modo de producción reproductivo», un modo no alejado del sistema esclavista, al cambiar dinero por personas. Junto con esto, critica como la cultura neoliberal y la economía de mercado imponen un proyecto de vida y forma de éxito basada en tener deseos y satisfacerlos, como el retrasar la maternidad con la congelación de óvulos o hacer uso de la gestación subrogada. Esto produce:

Unos deseos que el mercado convierte en derechos (del consumidor o del cliente) y que únicamente se encuentran sometidos a los límites legales existentes y a la capacidad económica de cada cual para asumir su coste. Todo ello en un contexto en el que el precio y el dinero [...] parecen liberar al consumidor/a de cualquier responsabilidad ética o moral (Nuño, 2016: 684).

En definitiva, la lectura de fondo de esta nueva iniciativa -junto a otras ya citadas como la gestación subrogada o como pudiera ser la venta y donación de óvulos- es otra manera más de control de las mujeres y de sus cuerpos, una mercantilización de éstos en el ámbito laboral mediante una iniciativa en la que el capitalismo y el patriarcado se dan la mano otra vez.

\section{Efectos de la maternidad para las mujeres en el ámbito empresarial}

En Estados Unidos ya se cuenta de manera pionera con empresas que retrasan la maternidad y paternidad por causas sociales -es decir, no motivadas por causas 
médicas ni enfermedades- por medio de diversas técnicas para una futura reproducción asistida. Concretamente, Prelude Fertility ${ }^{5}$ es una compañía fundada para crear un banco de óvulos y esperma para profesionales que están en la veintena y que se plantean aplazar la maternidad y la paternidad, pero no quieren sentirse condicionados por la calidad de su material reproductor en función de la edad en la que decidan tener hijos e hijas ${ }^{6}$.

Por su parte, según datos del IVI (2017) la preservación de óvulos en España por motivos sociales ha aumentado un $256 \%$ en los últimos cinco años, frente al $125 \%$ que obedece a motivos médicos. Además, señalan que el $84 \%$ de las mujeres que realizan tratamientos en esta institución no lo hacen por razones de enfermedad que les pueda impedir una futura maternidad, rondan los 37 años de media cuando optan por la vitrificación de ovocitos y sobre el $80 \%$ tiene estudios universitarios y principalmente se concentran en las áreas de la docencia, abogacía, medicina, economía y administración.

Así, Remohí afirma que la congelación de óvulos se concibe como «un seguro de vida. Si en los años 50 la píldora anticonceptiva fue una revolución sexual para la mujer, darle libertad de maternidad es una revolución social» (Europa Press, 2017).

Volviendo a la maternidad como freno para el desarrollo laboral, el estudio $\mathrm{Ma}$ ternidad y trayectoria profesional -realizado por el IESE Business School de la Universidad de Navarra- recoge que el 56\% de las mujeres españolas considera que tener hijos e hijas limita su trayectoria profesional, el 48,17\% reconoce haber esperado tener una posición laboral más segura y consolidada para tenerlos y a más del 50\% de las futuras empleadas se les ha preguntado en las entrevistas de trabajo si tienen o quieren tener hijos e hijas (Chinchilla, Jiménez y Grau, 2017: 42-45).

Esther Jiménez (2018) -decana de la Facultad de Educación de la Universidad de Cataluña y una de las autoras del anteriormente citado estudio Maternidad y trayectoria profesional-considera que con este tipo de medidas «vamos a un suicidio demográfico e iniciativas de este tipo se venden como un beneficio social, cuando en realidad es un beneficio para la empresa, que no ve interrumpida la productividad de sus trabajadores».

Y es que, las mujeres que también son madres -en comparación a otras que no lo son y a los hombres- son percibidas en las empresas como menos competentes, menos comprometidas con el trabajo remunerado, menos adecuadas para la promoción y, por tanto, merecedoras de salarios más bajos, ya que se las juzga de una manera más severa (Correll, 2007).

Desde la óptica de los feminismos, han sido diversas las visiones de la maternidad. De Beauvoir (1987) la consideraba un obstáculo para que la mujer lograra la igualdad, Firestone (1970) con el feminismo radical abogaba por que la función reproductiva se haga mediante técnicas donde no será necesaria la intervención de la

5 Disponible en: https://preludefertility.com/

6 Así, esta iniciativa se ve apoyada por datos como que en el $33 \%$ de las parejas que quieren tener hijos e hijas a partir de los 35 años sufren de infertilidad uno o ambos miembros, que el $50 \%$ de las mujeres embarazadas mayores de 45 años sufren abortos espontáneos y que el $80 \%$ de los embriones en esta franja de edad poseen anormalidades cromosómicas. 
mujer. Mitchell (1982) desde el feminismo del psicoanálisis defendía que el Estado asumiera la crianza y la educación de los hijos e hijas, ya que percibían que era un freno para la liberación de la mujer. Con el feminismo de la diferencia se reivindica la maternidad como fuente e identidad, señalando que el problema no es la maternidad en sí misma sino los efectos de desigualdad y opresión que genera asignar la responsabilidad exclusiva a las mujeres del cuidado de los hijos e hijas y del hogar, separa la dimensión biológica de la maternidad de lo social y trata de deconstruir la imagen creada y mantenida por el patriarcado.

El que la maternidad sea fruto de la división sexual de la función reproductiva -que corresponde biológicamente a la mujer- ha favorecido su construcción esencialista, universal, natural e inmutable, un patrimonio exclusivo de las mujeres, por lo que Solé y Parella (2004: 73) resaltan que «nos encontramos ante una compleja ideología funcional para los grupos dominantes, orientada a mantener la dominación masculina (patriarcado) y el sistema económico de explotación (capitalista)». Así, se asegura el cuidado de la futura fuerza de trabajo por parte de la mujer a un coste mínimo y, al mismo tiempo, hace posible contar con una reserva de fuerza de trabajo permanente. El capitalismo, pues, concentra la producción fuera del hogar y las mujeres pasan no sólo a producir sino a reproducir.

De esta manera se contribuye en el ámbito empresarial a la construcción de una segregación vertical o techo de cristal $^{7}$-obstáculos a los que se enfrentan las mujeres que aspiran a ejercer altos cargos en las organizaciones en igualdad de condiciones y salarios- y otra horizontal con la pared de cristal, que se evidencia a través de la calificación de masculino y femenino como características atribuidas a determinados trabajos (Barberá et al., 2002: 134).

Además, se completa con la asunción de las tareas de cuidado y vida familiar a la que tradicionalmente se relega a las mujeres como espacio supuestamente natural, por lo que el desarrollo profesional de las mujeres es alterado por las dobles jornadas y la falta de conciliación y corresponsabilidad por parte de las instituciones y de los varones -el llamado suelo pegajoso- lo que según Conde (2016: 20) dificulta el mantenimiento del equilibro entre trabajo dentro y fuera de casa y supone una sobrecarga de las vidas de éstas a través de los lazos afectivos y de responsabilidad adquirida y atribuida. Como consecuencia, comporta numerosos conflictos a nivel personal, familiar, social y también laboral. Concretamente, en el ámbito del trabajo se ven disminuidas las posibilidades de ascenso para las mujeres dentro de una empresa u organización «por tener que dedicar más tiempo a las tareas familiares y domésticas, ya sean una obligación social y familiar, o autoimpuesta desde los estereotipos y expectativas que tienen sobre ellas mismas en el ámbito privado».

Todo esto conlleva que muchas prefieran asumir puestos con escasas responsabilidades para no sobrecargarse, con escasas posibilidades de promoción, a media jornada o incluso que acaben abandonando sus puestos de trabajo y, por otro lado, que las propias empresas acaben seleccionando hombres en vez de mujeres, al tener más disponibilidad para el desarrollo de las responsabilidades. En España, la pre-

7 Acuñado en 1986 en un informe sobre mujeres ejecutivas: The Glass Ceiling- Special Report on the Corporate Woman (Hymowitz y Schellhardt, 1986). 
sidenta de la Fundación Mujeres, Marisa Soleto, subraya en una entrevista que el modelo general para poder trabajar y ser madre pasa por retrasar la maternidad y reducir el número de hijos e hijas, porque no se promueven otros mecanismos para compatibilizar, ya que desde la política o programas públicos no se está haciendo nada realmente eficaz y esa desigualdad laboral se refleja en los datos y agrega que:

Lo que necesitamos es que la capacidad organizativa de las empresas esté dentro de unas políticas públicas regidas por la idea de que maternidad y desarrollo profesional no son incompatibles [...]. Lo deseable sería plantearnos en qué estado están las bases de nuestros programas públicos para que las mujeres, si quieren ser madres, tengan que congelar sus óvulos o quedarse embarazadas a una edad en la que el coste sanitario y personal es más elevado (Pérez Mendoza, 2014).

Y es que las políticas y estructuras de las organizaciones empresariales están diseñadas frecuentemente para que el periodo entre los 30 y los 40 años de edad sea el más importante para el desarrollo de una carrera, que precisamente la etapa que exige una dedicación más intensiva en el cuidado de los hijos e hijas. Este contexto refleja una cultura organizacional en la que predominan los valores masculinos y las creencias estereotipadas de género, donde las mujeres sin responsabilidades familiares son vistas como madres potenciales (Agut y Martin, 2007: 206).

La profesora de Derecho del Trabajo de la Universitat de València y realizadora del citado estudio Maternidad y trayectoria profesional, Gemma Fabregat, considera que la congelación de óvulos no es positiva, sino que supone un retroceso en materia de igualdad, una intromisión en la intimidad de la trabajadora y de diferenciación entre unos y otros, que considera contraria a la Constitución y no debería incluirse en los convenios laborales: «Da igual lo que te digan. Te condicionan sí o sí porque hay un momento en el que tienes que compartir tu vida privada con la empresa. Y entonces pueden preparar una batería de medidas encaminadas a tu despido», subraya al periódico El Mundo (Lidón, 2018). Es decir, este tipo de medidas perpetúa de fondo la idea de que los cuidados no son compartidos, sino que corresponde a las mujeres, ya que no se fomenta su reparto.

Otro aspecto que contribuye al retraso de la maternidad en el ámbito empresarial es el riesgo de sufrir el llamado mobbing maternal ${ }^{8}$, por el que la mujer embarazada sufre la presión por parte del empleador con un objetivo ejemplarizante, es decir, como advertencia de los efectos que causa este «error», con graves consecuencias físicas y psicológicas para la afectada. Es un acoso que atenta contra la libertad de elección de las mujeres que ven peligrar sus puestos de trabajo y que tiene efectos graves sobre su salud y la de su futuro bebé.

En el estudio Cisneros XI Liderazgo Tóxico y Mobbing en la crisis económica (2009) -realizado por el Instituto de Innovación Educativa y Desarrollo Directivo- se señala como el 18\% de las trabajadoras denuncia que su organización presiona a las

8 Disponible en: http://www.elmobbing.com/mobbing-maternal-acoso-laboral (Fecha de consulta: $17 / 03 / 18)$. 
mujeres por causa de la maternidad y el $8 \%$ de las empleadas acosadas señala como principal causa el mobbing maternal y el $16 \%$ por razón de género, respectivamente.

Así que la maternidad tardía solo retrasa el problema laboral, porque el hecho de tener hijos e hijas -aunque sea a partir de los 40 años de edad- hace que sea un problema para la empresa. Con la congelación de óvulos se crea una medida preventiva para comprar tiempo y conseguir una mejor salud del embrión, pero el tiempo no se para en el cuerpo de la mujer y hay más riesgo de complicaciones en los embarazos más tardíos y el proceso es agresivo, tanto en la obtención -ya que hay que forzar la ovulación con diversa hormonas y medicamentos para acceder al ovario para tomar los óvulos- como para su posterior implantación en el útero, ya que la fertilización in vitro tiene un éxito de del $40 \%$ en mujeres menores de 35 años y del 15\% en las de 40, según señala la Sociedad Española de Fertilidad (SEF, 2011).

\section{Dificultades y soluciones para favorecer la conciliación}

La Ley Orgánica 3/2007, para la igualdad efectiva de mujeres y hombres, apareció como un instrumento legislativo que busca favorecer las condiciones de la mujer mediante la prevención y eliminación de conductas que la minusvaloren y excluyan. Es cierto que, hasta la creación de esta norma, los avances que se habían logrado hasta el momento se habían basado en la mujer y la maternidad como eje central de protección, pero con la aparición de esta jurisprudencia estatal se busca romper con el rol femenino y estereotipado de la mujer, a la que se le asocian las responsabilidades y los cuidados familiares.

Para ello, el fomento de la igualdad y la no discriminación de sexo son bases fundamentales para lograr un reparto equilibrado en las cargas familiares y un cambio en las actuales dinámicas empresariales, que impiden una mejor conciliación para todas y todos sus trabajadores. Pero, a diferencia de otros países europeos, en España aún no hay una política laboral consolidada que facilite de manera real una conciliación efectiva de la vida laboral y familiar, que se traduzca en «ajustar las necesidades personales y las laborales sin que una exceda a la otra, manteniéndolas proporcionalmente iguales y esto hoy en día aún es una utopía» (Agut y Martín, 2007: 206), ya que el papel que se adjudica a las mujeres de ser las principales cuidadoras del hogar y de la familia es lo que permite explicar la menor presencia femenina en la esfera pública en general, y en los puestos de trabajo de mayor responsabilidad en particular. Solé y Parella afirman que:

Se han dado unos cambios estructurales que permiten a la mujer incorporarse a la esfera pública, pero que «no se corresponden con cambios simbólicos alrededor de la maternidad «ideal», puesto que sigue vigente la inercia del modelo tradicional de la maternidad «intensiva». Este hecho permite explicar la inhibición en el número de hijos y la discordancia entre deseos y realidad (2004: 78).

En este sentido, Tobío resalta que -pese a una aceptación amplia del empleo femenino como positivo e incluso necesario- no hay conciencia de que las tareas 
de cuidado que han recaído siempre en las mujeres, tales como el cuidado de mayores, del hogar e hijos e hijas, deban organizarse de otra manera (Tobío, 2006: 25) porque «las mujeres están asumiendo nuevos roles laborales, pero siguen siendo las principales responsables del mundo doméstico. Tal superposición de funciones se percibe frecuentemente por parte de quienes la experimentan de forma más aguda, las madres que trabajan, como estar viviendo algo imposible» (Tobío, 2006: 25).

Por tanto, el rápido incremento del empleo femenino que se produjo en la década de los noventa en toda Europa «no vino impulsado por la extensión de las políticas de cuidado, sino más bien por la creciente demanda de fuerza de trabajo» (Tobío, 2006: 29). Esto ha creado tensiones y contradicciones entre la familia y el empleo, ya que las viejas responsabilidades domésticas se han superpuesto a las nuevas en el plano laboral «sin que la familia y la organización social hayan experimentado grandes cambios. Ello se traduce en un aumento del tiempo que las mujeres dedican a ambos tipos de trabajo, al doméstico y al remunerado o extradoméstico» (Tobío, 2006: 26).

En este contexto de escasas políticas sociales, poca implicación de los hombres en las tareas del hogar y de cuidados y la generalización del empleo a tiempo completo, Tobío (2006: 27-28) resalta como las madres españolas están desarrollando estrategias privadas e informales. Estas iniciativas se basan principalmente en procesos de sustitución y delegación de unas mujeres por otras como las abuelas o a través del mercado -que suelen ser mujeres inmigrantes cuyos hijos son cuidados en la distancia a su vez por otros familiares-, estableciéndose una cadena global de cuidados. Ante esto, Tobío considera que la práctica de estas acciones «quizá estén retrasando la toma de conciencia por parte del conjunto de la sociedad acerca de un problema social importante que a todos concierne» (2006: 27).

De acuerdo al estudio Mind the gap: Spain: Employee perspective (Grau-Grau, 2010) -realizado por Grau-Grau para conocer el punto de vista de las personas trabajadoras en España sobre la calidad laboral en sus puestos de trabajo-, la principal dificultad para favorecer la conciliación es la rigidez horaria, ya que el 70\% de los empleados tienen un horario fijo de entrada y salida (Grau-Grau, 2010: 10) pero, en el caso de tener las empresas políticas de flexibilidad, parecen no ser bien vistas. Así, el informe Maternidad y trayectoria profesional señala que pese a que el $62,49 \%$ de las mujeres de su muestra trabaja en empresas que tienen políticas de flexibilidad, el $64,5 \%$ percibe que «acceder a ellas conlleva consecuencias negativas para sus carreras».

En esta línea, Pasamar y Valle (2011: 21) señalaban en otro informe sobre las empresas analizadas del sector de alimentación y bebida que, casi dos terceras partes de los y las encuestados, consideraban que los directivos de su empresa no eran ejemplo de conciliación, lo que podía transmitir el mensaje de que el disfrute de estas medidas es incompatible con ascensos o promociones y podría aportar consecuencias negativas la carrera profesional de los trabajadores. Así, era llamativo el bajo porcentaje de uso de la mayoría de medidas de conciliación con las que contaban las organizaciones empresariales estudiadas. 
La segunda traba recogida en el estudio Maternidad y trayectoria profesional es la escasa sensibilidad en la cultura empresarial hacia la maternidad y paternidad. Concretamente, en el caso de las mujeres de la muestra de este informe, el 78\% considera que tiene pocas oportunidades profesionales para promocionar frente a los hombres y el 53\% señala que el hecho de ser madre ha sido una de las principales causas (2017: 48).

Dentro de las barreras sociopolíticas (2017: 49-50), tanto hombres como mujeres destacan principalmente las escasas ayudas a las familias y la maternidad y paternidad, a lo que se suma los estereotipos de roles femeninos y masculinos existentes y una concepción del éxito vinculado a lo masculino. A estos elementos también se añaden otros que no favorecen la conciliación y la corresponsabilidad y por los que se debería hacer una clara apuesta por cambiarlos, como son el reajuste entre los horarios y el calendario escolar y laboral -el 92\% de las mujeres y el 88,4\% de los hombres- o los horarios de comida extensos y tardíos -el 77.5\% de las mujeres y el $73,9 \%$ de los hombres, respectivamente. Ante este panorama, Chinchilla, Jiménez y Grau resaltan que:

A pesar de ser la familia la institución más valorada por los españoles, existe una percepción mayoritaria del escaso reconocimiento que se presta en España a las familias. Y esto tiene mucho que ver con la escasez de ayudas y una visión distorsionada del papel que desempeña.

El reconocimiento es distinción y, es a la vez, gratitud. Una manera de agradecer y distinguir el papel social de la maternidad, de la paternidad y de la familia es otorgarle el valor que realmente tiene en la sociedad con medidas que lo avalen. Una de las muchas maneras que tienen los gobiernos de demostrar fácilmente la prioridad que conceden a esta institución social, es dotarla de los necesarios recursos, ampararla mediante leyes oportunas, e institucionalizar un tema que, más que nunca, se ha vuelto prioritario (Chinchilla, Jiménez y Grau, 2017: 50).

También hay que resaltar las barreras familiares (2017: 51-52), dentro del espacio privado, que se traducen en una falta de apoyo o desigualdad. En este sentido, los porcentajes entre mujeres y hombres son similares y el orden de factores también, aunque con matices. $Y$ es que si bien ambos destacan la falta de flexibilidad del cónyuge como el primer obstáculo familiar para favorecer la conciliación y corresponsabilidad en porcentaje similar -algo más del 70\% para ambos sexos-, la diferencia se acentúa cuando se habla de las siguientes causas: como son la sobrecarga de roles -el 60,4\% de las mujeres y 47,6\% de los hombres-, o el poco apoyo de la pareja en casa, con el 68,6\% de las mujeres y el 53\% de los hombres.

No hay que olvidar las barreras personales (2017: 52-53), ya que los roles y estereotipos vienen fijados por patrones construidos y naturalizados, muchas veces aprehendidos de manera inconsciente, fruto de los diferentes agentes -familiares, sociales, educativos, sanitarios, legislativos- que conforman e influyen en las personas. La falta de formación en áreas tradicionalmente masculinas -como la ingeniería, ciencia, tecnología y matemática- en un 54\%, seguidas de la infravaloración, el miedo al fracaso y la falta de confianza en sí misma -con el 34,8\%,34,5\% y 28,4\%, 
respectivamente- son las principales razones que las mujeres esgrimen como obstáculos en su posibilidad de acceso y promoción en el mundo laboral.

La falta de confianza retroalimenta la metáfora de la profecía autocumplida, con la que se explican las formas en las que la cultura organizacional de las empresas frena el desarrollo psíquico y social de las mujeres. Frente a esto, Barberá y otros abogan por difundir lo positivo y valioso que aportan las mujeres para el valor y entorno empresarial, por lo que:

Hay que contribuir a transformar la creencia popular que sostiene que las mujeres no tienen motivación de poder por nuevas representaciones que realcen el valor social de algunos atributos femeninos en los que históricamente se ha socializado a las mujeres (Barberá et al., 2002: 12).

Ante esto, Gimeno y Rocabert consideran clave para aumentar la autoconfianza de las mujeres que cambien sus estilos de atribución para que,

Sus éxitos dejen de ser atribuidos siempre a causas internes inestables (esfuerzo) y comiencen a atribuirlos, como hacen generalmente los hombres, a causas internas estables (inteligencia), junto con la atribución de algunos casos de sus fracasos a casos externas (dificultad de la materia, mala suerte) o a causas internas inestables (falta de esfuerzo) (Gimeno y Rocabert, 1998: 32).

Además, se observa en muchas ocasiones como la mujer es un obstáculo para sí misma al mostrar una actitud de ambivalencia que desarrolla en su discurso de la igualdad y la maternidad como un espacio propio que se le atribuye exclusivamente a ella, fruto de la socialización diferenciada de género, que está impregnada de la maternidad intensiva. Concretamente, reclama la igualdad total entre hombres y mujeres en la esfera pública y en la privada desde la racionalidad pero, por otro lado, muestra un sentimiento de culpa por el hecho de no poder proporcionar una presencia a sus hijos e hijas que no exigen o no esperan de los hombres (Solé y Parella, 2004: 86).

Por tanto, se aprecia como en España -al igual que ocurre en otros Estados del Bienestar- hay falta de apoyo institucional para conciliar la vida laboral y familiar, un elemento más para disuadir a las mujeres que participen en el mercado laboral y que se dediquen a la maternidad. En este sentido, León subraya que:

Las mujeres que prevén un alto conflicto entre la esfera laboral y la vida familiar, o bien es menos probable que trabajen, o bien acaban «resolviendo» el conflicto renunciando a tener hijos. Así, lo que Hobson y Oláh (2006) han llamado «la huelga de la fecundidad», es más probable que se produzca en países con débiles políticas de conciliación, incluyendo el cuidado infantil [...]. De esta manera, la perspectiva de Inversión Social argumenta que facilitar que las mujeres puedan conciliar su vida familiar con su participación en el mercado laboral tiene resultados positivos en la fertilidad y la productividad económica en general (León, 2015: 33). 
Así, en el contexto empresarial español, sumado a los factores sociales y personales, hacen que, tal y como señala Chinchilla, Jiménez y Grau, la población padezca:

Un invierno demográfico, por la persistente baja tasa de fertilidad. Sin embargo, existe una clara diferencia entre el número de hijos reales (1.32), el número de hijos esperados (1.69), y el número de hijos deseados (2.52). Los datos muestran que tanto mujeres como hombres deben renunciar al número de hijos que desearían tener, por los obstáculos que encuentran fundamentalmente en la sociedad y en la empresa, así como por la falta de apoyo de los gobiernos (Chinchilla, Jiménez y Grau, 2017: 55).

Por ello, Tobío resalta la necesidad de abordar la conciliación desde una perspectiva integral, mediante la definición de una modalidad de articulación familiaempleo, la puesta en marcha de políticas concretas que la desarrollen y las transformaciones en las relaciones de género y en la familia, ya que estas últimas son «a la vez causa y efecto de la asunción de nuevas responsabilidades de aportación económica por parte de las mujeres. Desean asumirlas, pero además, la economía y la sociedad necesitan que lo hagan» (Tobío, 2006: 32).

\section{Conclusiones}

Ante el panorama anteriormente descrito, Tobío subraya como «vivimos hoy en una inercia de viejos modelos que se superpone a nuevas pautas, generando tensiones difícilmente sostenibles. Es tiempo ya de construir un nuevo modelo de relación entre familia y empleo» (Tobío, 2006: 32).

Si bien es cierto que es complicado tratar de legislar el espacio privado-donde el empoderamiento de las mujeres, la implicación de sus parejas en materia de corresponsabilidad y conciliación para todas y todos y la educación de sus hijos e hijas son clave-, sí se podría tratar de aplicar medidas en el espacio de lo público y desde lo público, tales como el establecimiento de horarios más racionales, con el reajuste del horario escolar compatible con el laboral y favorecer en los concursos públicos a aquellas empresa que aplican y promueven medidas que favorecen la conciliación. También se debería apostar por revalorizar el papel de las familias mediante campañas de concienciación y realizar una mayor inversión en la familia, ya que -según el informe Evolución de la familia en España 2016 realizado por el Instituto de Política Familiar, nuestro país destina el 1,4\% del PIB para apoyar a las familias frente al 2,2\% de media en la Unión Europea.

Otras propuestas de mejora podrían pasar por reformar aspectos de la Seguridad Social con los que se eviten sanciones por el ejercicio de la maternidad y la paternidad, como son la ampliación de la baja en un año y la garantía de volver al trabajo con formación desgravable que facilite su reincorporación, cubrir la cotización de los periodos de excedencia por maternidad o paternidad o introducir en las cuentas públicas el valor del trabajo doméstico no remunerado -siendo ése último punto un debate que ya se arrastra desde la década de los 70-. Además, la empresa debe favorecer medidas flexibles en el tiempo y el espacio y la política de sustitu- 
ciones durante el tiempo de baja de maternidad o paternidad, crear una cultura a favor de las familias y aplicar acciones que no penalicen la maternidad dentro del desarrollo profesional en las empresas como son los currículums ciegos, la formación en materia de género, la presencia de mujeres en los puestos de dirección o la creación de programas específicos para identificar, desarrollar y promocionar empleadas con gran potencial (Chinchilla, Jiménez y Grau, 2017).

Por ello, para promover la natalidad, la sociedad debe promover acciones estructurales que garanticen y favorezcan la crianza y las empresas han de evitar la discriminación laboral de la mujer con medidas de corresponsabilidad en la crianza y de flexibilización. Esto permitiría que las mujeres decidieran, dentro de políticas integrales de conciliación, si quieren retrasar o no el momento de ser madres y, por otra parte, no sería necesario contar por parte del sector económico con acciones que inviten a retrasar y mercantilizar la maternidad y, en definitiva, a coartar los cuerpos de las mujeres.

\section{REFERENCIAS BIBLIOGRÁFICAS}

Agut Nieto, Sonia y Pilar Martín Hernández (2007): «Factores que dificultan el acceso de las mujeres a puestos de responsabilidad: una revisión teórica» en Apuntes de Psicología, Vol. 25, No2, pp. 201-214.

Barberá Heredia, Esther; Ramos, Amparo; Sarrió, Maite y Carlos Candela (2002): «Más allá del techo de cristal. Diversidad de género» en Revista del Ministerio de Trabajo y Asuntos Sociales, N40, pp. 55-68.

Chinchilla, Nuria; Jiménez, Esther y Marc GRAu (2017): Maternidad y trayectoria profesional en España. Análisis de las barreras e impulsores para la maternidad de las mujeres españolas, Navarra: IESE Business School. Disponible en: http:/ /www. iese.edu/Aplicaciones/upload/IESEORDESAlow.pdf (Fecha de consulta: 16/03/18).

Correll, Shelley J. y Stephen Bernard (2007): «Getting a Job: Is there a motherhood penalty?» en American Journal of Sociology, Vol. 112, No5, pp. 1297-1338.

De Beauvorr, Simone (1972): El segundo sexo, Buenos Aires: Siglo XX.

Europa Press (2017): "Trabajadores de empresas valencianas que firmen un acuerdo con el IVI tendrán mejores condiciones para preservar óvulos» en Europapress.es. Disponible en: http://www.europapress.es/comunitatvalenciana/noticia-trabajadores-empresas-valencianas-firmen-acuerdo-ivitendran-mejores-condiciones-preservar-ovulos-20171114132116.html (Fecha de consulta: 13/03/18).

Firestone, Shulamith (1976): La dialéctica del sexo, Barcelona: Kairós.

GIMENO, M J José y Esperanza RocABERT (1998): «Barreras percibidas por las mujeres en su proceso de toma de decisión vocacional» en ROP, Vol. 9, No 15 , pp. 25-36.

GobieRno de EsPAÑA (2007): Ley Orgánica 3/2007, de 22 de marzo, para la igualdad efectiva de mujeres y hombres. Disponible en: http://www.empleo.gob.es/es/ publica/pub_electronicas/destacadas/revista/numeros/ExtraIgualdad07/ Docu01.pdf (Fecha de consulta: 19/03/18). 
Grau-Grau, Marc (2010): Mind the gap: Spain: Employee perspective, Boston: The Sloan Center of Ageing and Work. Disponible en: https://www.bc.edu/ content/dam/files/research_sites/agingandwork/pdf/publications/MTG_ Spain_Employee.pdf (Fecha de consulta: 10/08/18).

Guerra Palmero, María José (2017): «Contra la mercantilización de los cuerpos de las mujeres. La "gestación subrogada" como nuevo negocio transnacional» en Dilemata. Revista Internacional de Eticas Aplicadas, No26, pp. 39-51.

Instituto de InNovación Educativa y Desarrollo Directivo (2009): Barómetro CISNEROS XI. Liderazgo Tóxico y Mobbing en la crisis económica. Resumen de datos preliminares. Disponible en: http://www.cincodias.com/5diasmedia/ cincodias/media/200907/02/economia/20090702cdscdseco_2_Pes_PDF.pdf (Fecha de consulta: 18/03/18).

Instituto DE Política FAmiliar (2016): Informe Evolución de la familia en España 2016. Disponible en: https://www.observatoriodelainfancia.es/ ficherosoia/documentos/4929_d_Informe_Evolucion_de_la_Familia_\%20en_ Espa\%C3\%B1a_2016_c.pdf (Fecha de consulta: 18/03/18).

Instituto Nacional de Estadística (2017): Cifras de Población a 1 de julio de 2017 Estadística de Migraciones. Primer semestre de 2017. Datos Provisionales. Disponible en: http:/ / www.ine.es/prensa/cp_j2017_p.pdf (Fecha de consulta: 16/03/18).

Instituto Nacional de Estadística (2017): Indicadores de Fecundidad. Resultados nacionales. Edad Media a la Maternidad por orden del nacimiento según nacionalidad (española/extranjera) de la madre. Disponible en: http://www.ine.es/jaxiT3/ Tabla.htm?t=1579\&L=0 (Fecha de consulta: 17/07/18).

INSTITUTO VALENCIANO DE INFERTILIDAD (2017): «Los tratamientos para preservar la fertilidad en IVI crecen por encima del 250\%» en Ivi.es. Disponible en: https: / / ivi.es/blog/los-tratamientos-para-preservar-la-fertilidad-en-ivi-crecen-porencima-del-250/ (Fecha de consulta: 15/03/18).

LEÓN BorJA, Margarita (ed.) (2016): Empleo y maternidad: obstáculos y desafíos a la conciliación de la vida laboral y familiar, Barcelona: Universitat Autònoma de Barcelona. Disponible en: http://igop.uab.cat/wp-content/uploads/2016/01/ Informe-FUNCAS-IGOP.pdf (Fecha de consulta: 19/3/18).

LidóN, Inma (2018): «La congelación de óvulos en la empresa llega a España: ¿beneficio laboral o abuso?» en El Mundo. Disponible en: http: / / www.elmundo. es/papel/historias/2018/01/08/5a52647ee2704eda078b4621.html (Fecha de consulta: 15/03/18).

Mitchell, Juliet (1976): Psicoanálisis y feminismo, Barcelona: Anagrama.

Nuño GómEz, Laura (2016): «Una nueva cláusula del Contrato Sexual: el vientre de alquiler» en Isegoría. Revista de Filosofía Moral y Política, No55, pp. 683-700.

PASAMAR, Susana y Ramón VAlle (2011): «Conciliación de la vida profesionalpersonal en las empresas españolas. ¿Mito o realidad?» en Universia Business Review, 1er. trimestre, pp. 14-31.

Pérez Mendoza, Sofía (2014): «Retrasar la maternidad para promocionar en el trabajo: ¿oportunidad o muestra de un sistema perverso?» en Grup de Dones de laMarxa Mundial València. Disponible en: https://www.nodo50. 
org/xarxafeministapv/?+Retrasar-la-maternidad-para (Fecha de consulta: $17 / 03 / 18)$.

SOCIEDAd EsPañola de FERTILIDAd (2011): Saber más sobre fertilidad y reproducción asistida. Disponible en: http://www.sefertilidad.net/docs/pacientes/spr_sef_ fertilidad.pdf (Fecha de consulta: 17/03/18).

SolÉ, Carlota y Sonia Parella (2004): «Nuevas» expresiones de la maternidad. Las madres con carreras profesionales «exitosas» en Revista de Estudios Sociológicos, $\mathrm{N}^{\circ} 4$, pp. 67-92.

Toвío Soler, Constanza (2006): «Nuevas formas familiares: las madres que trabajan» en Pasajes: Revista de pensamiento contemporáneo, No19, pp. 25-32.

Recibido el 30 de marzo de 2018

Aceptado el 23 de julio de 2018 BIBLID [1132-8231 (2018): 45-59] 